\title{
Meningitis bacteriana: Identificación del agente causal por aglutinación de látex
}

\author{
Dr. Rober to Bustos L. ${ }^{1}$; Dra. M. Teresa Broussajn B. ${ }^{2}$; T.M. Angela Tejero P. ${ }^{2}$; \\ Dr. Richard Ríos R. ${ }^{1}$; Dr. Jaime Jara $S^{3}$ \\ Acute bacterial meningitis: \\ etiologic diagnosis by latex aglutination
}

Findings at bacterial cultures and CSF latex agglutination test in $\mathbf{4 8}$ children with acute bacterial meningitis are described. Bacterial isolates were obtained from CSF cultures in 31/48 cases (64.6\%). Latex agglutination tests were performed in $44 / 48$ patients and gave positive results in $32 / 44(72.7 \%$, allowing identification of causative agents in 8 patients whose cultures were negative. Haemophilus influenzae was the most prevalent agent $(61.5 \%)$ with preference for infants aged three to welve months. Antibiotic sensitivity was tested by minimal inhibitory concentrations (MIN). Three strains of $H$. infiuenzae were sinultaneously resistant to both ampicillin and chloramphericol, whereas all tested strains were susceptible to cefotaxime. Other indentified bacteriae were Streptococcts pneumoniae $(20.5 \%)$ and Neisseria meningitidis $(12.8 \%)$.

(Key words: meningitis, acute, bacterial, etiologic diagnosis, la tex agglutination test).

La identificación de los diferentes agentes causales de meningitis bacteriana aguđa (MBA), es de importancia fundamental por sus implica-

1. Instituto de Pediatría, Universidad Austral de Chile.

2. Instituto Microbjologia Clínica, Universidad Austral de Chile.

3. Servicio de Pediatıía, Hospital de Valdivia.

Proyecto S-84-16 DID, Universidad Austral de Chile. ciones terapéuticas, pronósticas y epidemiológicas. La mayor parte de los casos en lactantes y preescolares, son provocados por Haemophitus influenzae, Streptococcus pneumoniae o Neisseria meningitidis, correspondiendo a $\mathrm{H}$. influenzae tipo $b$, un rol prepondenrante. ${ }^{1-11}$

El diagnóstico etiológico de certeza se obtiene a través del cultivo bacteriano, que además permite el estudio de susceptibilidad a los antimicrobianos, lo cual es particularmente necesario si 
consideramos la aparición en nuestro país de cepas de $H$. influenzae resistentes a ampicili. na. $12 \cdot 14$

Uno de los factores que disminuye el rendimiento de la bacteriologia, es el tratamiento antibiótico previo, antecedente que observamos con frecuencia en nuestros pacientes, que en su mayoría proceden del área rural. El desarrołlo de técnicas de diagnóstico inmunológico basadas en la detección de ant f́genos bacterianos en el líquido cefalorraquídeo ( $\mathrm{LCR}$ ), que no requieren necesariamente de la presencia de bacterias viables, ha permitido una orientación etiológica pocos minutos después de ingresado el paciente, posibilitando además la jdentificación de gérmenes en afgunos-casos de cultivo negativo. Una de estas pruebas, consistente en la aglutinación de partículas de látex sensibilizadas, ${ }^{15 \cdot 24}$ se ha demostrado útil como examen complementario de diagnóstion, con las ventajas de ser de fácil ejecución e instalación.

Los propósitos de este estudio fueron obtener una visión actualizada de las diferentes etiologías de meningitis bacteriana aguda en nuestra área, analizar su susceptibilidad a los antimicrobianos y evaluar la utilidad de la prueba de aglutinación de látex como examen auxiliar.

\section{MATERIAL Y METODO}

Se estudiason, en forma prospectiva, 48 pacientes hospitalizados con diagnóstico de meningitis bacterians aguda, en la unidad de infecciosos del survicio de pediatria del Hospital de Valdivia, entre julio de $1984 \mathrm{y}$ julio de 1987. Il diagnóstico sc basó en las man ifestaciones clínicas, won o sin signos meníngeos $y$ las siguientes alteraciones del examen ciloquímico del LCR: flucosa inferior a $40 \mathrm{mg}^{\%}$ cetularidad superior a 50 por $\mathrm{mm}^{3}, y$ predominio de polinucleares. Sc descartaron los casos que plantearan dudas entre etiología bacteriana o viral.

Las 48 muestras de LCR, obtenidas al ingreso, se sometieron a estudio citoquímico, tinción de Gran $y$ cultivo; en 44 se realizó la prueba de aglutinación de látex. Jil cultivo se realizo ch agar sangre con satebtismo, agar chocolate y caldo tioglicolato, incubandose hasta 5 dias, cn almóslera de 5 a $10 \%$ de $\mathrm{CO}_{2}$.

E] estudio de susceptibilidad antimicrobiana en todas las cepas de Haemophilus influenzae assladas, se realizó mediante antibiograma por difusión, para ampicilina, cloramfenicol y cefotaxima $y$ en 7 cepas se investigó ademas ceftriaxona y cefuroxima. Se midió concentración inhibitoria mínima (CIM) en 13 cepas de $H$. infuenzae. para ampicilina, cloramfenicol $y$ cefotaxima. En 3 cepas de Neisseria meningitidis y 3 de Strepto. coccus pneumoniae, se determino CIM para penicilina. cloramfonicol, cefotaxima y ampicilina.

La determinación de CIM se realizó utilizando el método de Robson y Salit, 25 efectuando diluciones seriadas entre 256 y $0,03 \mu \mathrm{g} / \mathrm{mL}$ para penicilina, $64 \mathrm{y}$ $0,25 \mu \mathrm{g} / \mathrm{mL}$ para cloramfenicol, entre 32 y $0,03 \mu \mathrm{g} / \mathrm{mL}$ para cefotaxima y entre $256 y 0,03 \mathrm{Hg} / \mathrm{mL}$ para ampicilina.

La determinación de betalactamasas se realizó por et método de la cefalosporina cromógena. 29

Para la prueba de aglutinación de látex se utilizó ct "Slidex Meningite Kit" (Bio Merieux) que contiene partículas sensibilizadas a antigenos capsulares de $H$. influenzoe tipo b, S. pneumonia (83 serotipos) y $N$. meningitidis grupo A y C. [i] umbral de sensibilidad para $H$. influenzae tipo b y $N$. meningitidis $A$ y $C$ es de 0,025 jg/mL y para St. pneumoniae $0,1-0,5 \mathrm{~kg} / \mathrm{mL}$. La prueba fue ejecutada por el mismo operador, y sus resultados, de tipo cualjtatativo, se registraron en una escala de 4 cruces, de acuerdo a pauta de Newmsn. 19

\section{RESULTADOS}

En el período de estudio señalado, ingresaron 48 pacientes con diagnóstico de meningitis bacteriana aguda, 32 de sexo masculino $(66,6 \%)$ y 16 de sexo femenino $(33,3 \%)$. Las edades extre. mas fueron 17 días y 8 años; 34 pacientes eran menores de un año $(70,8 \%), 6$ tenían entre 1 y 2 años y 8 eran mayores de 2 años; 35 pacientes eran eutróficos, 9 sufrían desnutrición calórica proteica grado 1 (según Sempé), 2 desnutrición grado $\amalg, y$ en 2 no se consignó estado de la nutrición al ingresar. Sólo $25 \%$ de los pacientes provenian de la ciudad de Valdivia, el resto de localidades distantes.

Los cultivos de LCR fueron positivos en 31 casos $(64,6 \%)$ (tabla 1). La tinción de Gram fue concordante con el cultivo bacteriano en $93,5 \%$ (29/31), y fue negativa en dos casos de cultivos positivos para $N$. meningitidis y Staphilococcus aureus respectivamente. Los hemocultion, realizados en 33 pacientes, fueron positivos en sólo 2 $\operatorname{casos}(6 \%)$, aislándose $N$. meningitidis en uno y $H$. Influenzae en el otro.

La prueba de aglutinación de látex fue positiva en 32 de 44 pacientes $(72,7 \%)$. Las determj.

Tabla 1.

Cultivo $y$ tinción de Gram en 31 casos de meningitis bacteriana aguda

\begin{tabular}{lrrr}
\hline Gormen & No & $\%$ & Gram \\
\hline Haemophilus influenzac & 19 & 61,3 & 19 \\
Streptococcus pneumoniae & 6 & 19,3 & 6 \\
Neisser iz meningitidis & 4 & 12,9 & 3 \\
Streptococcus grupo B & 1 & 3,2 & 1 \\
Staphylococcus aureus & 1 & 3,2 & - \\
\hline \hline Total & 31 & & $29(93,5 \%)$ \\
\hline
\end{tabular}


naciones positivas correspondieron a $H$. influenzae tipo b: $\mathrm{n}=22$; St. pneumoniae: $\mathrm{n}=7$ y $N$. meningitidis grupo $\mathrm{C}: \mathrm{n}=3$. No se registraron falsos positivos. Se constataron 2 reacciones cru. zadas débiles: una entre $H$. influenzae $(2+)$ y $N$. meningitidis grupo $C(+)$ y otra entre $H$. influen. zae tipo $b(3+)$ y $N$. meningitidis $A$ y $C(+)$ tardía. En ambos casos el cultivo confirmó la presencia de $H$, influenzae.

En la tabla 2 se detallan los resultados de cultivo, Gram y látex, para los principales gérmenes encontrados.

E1 $H$. influenzae, se identificó por cultivo y látex, con tínción de Gram concordante en 17 pacientes. En otros 5 casos cuyo cultivo fue negativo, la identificación se logró con prueba de látex y tinción de Gram, 4 de ellos registraban antecedentes de tratamiento antibiótico previo. En 2 pacientes con cultivo LCR positivo a Haemophilus, la pr ueba de látex fue negativa. En base a estos antecedentes, la sensibilidad observada para esta prueba fue de $89,5 \%$ (17/19). La tinción de Gram fue concordante, en el 100\% con las determinaciones de látex positivas.

St. pneumoniae, se diagnosticó en 5 casos por cultivo y látex. En 2 pacientes, la identificación se logró sólo con prueba de látex y Gram, ambos con antecedentes de tratamiento previo con penicilina intramuscular por 2 días. Esta prueba (látex) se omitió en un caso, cuyo cultivo fue

Tabla 2 .

Resultados de Gram, cultivo y pruebas de látex

\begin{tabular}{|c|c|c|c|}
\hline & $\begin{array}{c}\text { H. } \\
\text { Infhuenzac }\end{array}$ & $\begin{array}{c}\text { St. } \\
\text { Pneumoniat }\end{array}$ & $\frac{N}{\text { Meningitikdis }}$ \\
\hline Grom, c ultivo y Látex $(+)$ & 17 & 5 & 2 \\
\hline Gram y Lálex (+) Cultivo (-) & 5 & 2 & t \\
\hline Gram y Cultivo (t) Litex (-) & 2 & - & 1 \\
\hline Gram y Cultivo (t) ( & - & 1 & - \\
\hline Sijlo (ultivo $(++14)$ & - & & $\mathbf{I}$ \\
\hline Tatal gérmenes ident jlicados & 24 & 8 & 5 \\
\hline
\end{tabular}

positivo. No se detectaron reacciones cruzadas.

Para $N$, meningitidis la prueba de látex fue positiva en 3 pacientes, todas del grupo $C_{\text {, en uno }}$ de los cuales, el cultivo correspondiente fue negativo (tratado con penicilina, cloxacilina y gentamicina i.m. un día antes). Sólo en una de las 4 cepas cultivadas se hizo serotipificación, correspondiendo ésta al grupo $\mathbf{C}$, en concordancia con látex. En un caso de cultivo positivo la prueba de látex fue negativa.

Para otros gérmenes cultivados: Streptococcus grupo $B$ en un lactante de un mes 14 dias de edad y Stophylococcus aureus en un recién nacido de 17 días, la prueba de látex fue negativa en ambos. En conjunto, esta reacción permitjó orientación etiológica en 8 casos cuyos cultivos fueron negativos, con correlación $100 \%$ con la tinción de Gram. De esta forma el diagnóstico etiológico se alcanzó en 39 casos $(81,2 \%)$ cuya distribución por grupos etarios se detalla en la tabla 3.

En conjunto los cultiwos de LCR fueron negativos en 17 pacientes $(35,4 \%)$, de los cuales 15 habían recibido tratamiento antibiótico previo. La prueba del látex realizada en 14 de estos casos, fue positiva en 8 y negativa en $6, y$ la tinción. de Gram fue negativa sólo en 6 pacientes de este grupo.

La susceptibilidad a los antimicrobianos se determinó mediante CIM para 13 cepas de $H$. influenzae para ampicilina, cloramfenicol y cefotaxima (tabla 4). Se identificaron 3 cepas resistentes simultáneamente a ampicilina y cloramfenicol, todos productoras de betalactamasas. Cefotaxima en cambio fue activa sobre todas las cepas estudiadas (tabla 5). El estudio de susceptibilidad por técnica de difusión en placa, fue concordante con los hallazgos obtenidos por técnica de dilución (CIM), comprobándose además susceptibilidad de las 7 cepas para las cuales se evaluó cefuroxima y ceftriaxona.

Tabla 3.

Etiología meningitis bacteriana aguda según grupo etario en 39 pacientes

\begin{tabular}{|c|c|c|c|c|c|c|c|}
\hline Germen & $\begin{array}{c}<3 \text { meses } \\
(N=512.8 \%)\end{array}$ & $\begin{array}{c}3-6 \text { meses } \\
(\mathrm{N}=1230.8 \%)\end{array}$ & $\begin{array}{c}7-12 \text { meses } \\
(N=1128.2 \%)\end{array}$ & $\begin{array}{l}13-24 \text { meses } \\
(\mathrm{N}=410.2 \%\end{array}$ & $\begin{array}{l}>24 \text { meses } \\
(\mathrm{N}=718 \%\end{array}$ & Total & $\%$ \\
\hline H. influenzae & - & 10 & 9 & 2 & 3 & 24 & 61,54 \\
\hline St. pneumoniae & 2 & 2 & 1 & 2 & 1 & 8 & 20,51 \\
\hline $\mathrm{N}$, meningitidis & 1 & - & 1 & - & 3 & 5 & 12,82 \\
\hline St. grupo B & 1 & - & - & - & - & 1 & 2,56 \\
\hline Staph. aureus & 1 & - & - & - & - & 1 & 2,56 \\
\hline
\end{tabular}


Tabla 4.

Susceptibilidad in vitro (CIM en $\mu \mathrm{g} / \mathrm{ml}$ ) de 13 cepas de $H$. Influenzae frente a 3 antibióticos

\begin{tabular}{lrcr}
\hline Antibjótico & $50 \%$ & $\begin{array}{c}\text { CIM } \\
90 \% / \mathrm{ml}\end{array}$ & \multicolumn{1}{c}{ Rango } \\
\hline Ampicilina & 0,25 & $\geqslant 256$ & $\leqslant 0,03-\geqslant 256$ \\
Choramfenicol & 1 & 16 & $0,5->64$ \\
Cefotaxima & $\leqslant 0,03$ & 0,5 & $\leqslant 0,03-1$ \\
\hline
\end{tabular}

Nivel de susceptibilidad 28; ampicilina $\leqslant 2$, cloramficniol $\leqslant 8$, cefotaxima $\leqslant 8$. CIM $50 \%$ y $90 \%$ Inhibiciod del $50 \%$ y $90 \%$ de las cepas respecturamente

Tabla 5 .

CIM $(\mu \mathrm{g} / \mathrm{ml})$ de 3 cepas de

H. Influenzae Multiresistentes

\begin{tabular}{lccc} 
& Ampicilina & Clisamfonicul & Cofotaxima \\
\hline CIPA 1 & $\geqslant 256$ & $\geqslant 64$ & 1 \\
ClPA 2 & $\geqslant 256$ & 16 & 0,12 \\
CIPA 3 & $\geqslant 256$ & 16 & $\leqslant 0,03$ \\
\hline \hline
\end{tabular}

Las 3 cepas de $\mathbf{N}$. meningitidis, en las que se determinó CWM para cefotaxima, penicilina, cloramfenicol y ampicilina, fueron altamente susceptibles, igual que 3 cepas de $S t$, pneumoniae frente a penicilina, cefotaxima y cloramfenicol.

\section{COMENTARIO}

En 39 de 48 pacientes estudiados se pudo precisar la etiologia por cultivo por prueba de látex, concordante $\infty$ la tinción Gram. $H$. Influenzae fue el agente etiológico más frecuente. $\mathrm{La}$ incidencia fue notoriamente inferior para $S t$. pneumoniae y $N$. meningitidis. Cabe destacar que $H$. influenzae no se identificó en menores de 3 meses de edad, pero en el grupo et ario entre 3 a 12 meses, con etiología demostrada, este germen fue responsable de la gran mayoria de los casos.

La escasa positividad de los hemocultivos (6\%) puede atribuirse presumiblemente a problemas de técrica.

La. prueba de aglutinación de látex fue positiva en una elevada propor ción $(72,7 \%)$ de las determinaciones. Si se excluyen los 2 pacientes con cultivo de LCR a Streptococcus grupo B y Staphylococcus aureus, para los cuales la prueba fue negativa, su rendiniento aumenta. Por otro lado la prueba fue negativa en 2 pacientes de cuyo LCR se aislaron $H$. influenzae y $N$. menin- gitidis respectivamente. Dado que no fue posible serotipificar esas cepas, no podemos asegurar si ese resultado correspondió a falso negativo o a serotipos de $H$. influenzae diferente al $b,{ }^{25}$ y de $N$. meningitidis distintos a grupos A y C; sólo se comprobaron 2 reacciones cruzadas débiles.

La sensibilidad del método de látex, para $H$. influenzae fue muy alta: consideramos que es un examen complementario de gran utilidad por ser sencillo, de costo razonable y no requerir equipamiento complejo. Obviamente no reemplaza a otros estudios microbiológicos, pero permite una orientación etiológica rápida inicial, Jo cual es importante desde el punto de vista terapéutico, pronóstico y epidemiológico.

La tinción de Gram mostró alto rendimiento y concordancia con los cultivos de LCR y reacciones de látex. En 2 lactantes en los que el único examen positivo fue la tinción de Gram negativa con bacilos pleomórficos, la meningitis se asoció clínicamente a celulitis facial, situación altamente sugerente de infección con $H$. influen$z a e^{2,3,5,7,9}$ En otros casos la tinción de Gram reveló diplococcus Gram negativos intra y extracelulares, sugerentes de $N$. meningitidis.

El estudio de susceptibilidad antimicrobiana detectó 3 cepas de $H$. influenzae resistentes simultáneamente a ampicilina y cloramfenicol. Dos de estos pacientes, de 3 y 5 meses de edad, fueron tratados con estos antibióticos y fallecieron antes de recibir el informe de sensibilidad. El tercero respondio satisfactoriamente a cefotaxima. La resistencia a ampicilina de estas 3 cepas, de un total de 19 cultivadas da una frecuencia del fenómeno de $15.7 \%$ en esta serie. Esta situación es agravada por el hecho de detectarse resistencia simultánea a cloramfenicol, ${ }^{13.27}$ y por tanto no se puede considerar a este antibiótico como alternativa confjable de tratamiento, mientras no se disponga del informe de susceptibilidad correspondiente. 12,13

Todas las cepas estudiadas fueron altamente susceptibles a cefotaxima como también a cefuroxima y ceftriaxona, por el método de difusión.

El panorama etiológico encontrado en este estudio, confirma la situación descrita a nivel internacional y en otros centros nacionales, respecto al rol preponderante que Haemophilus influenzae tipo b tiene actualmente en la menir. gitis bacteriana aguda en el niño, y enfatiza la necesidad de contar con una vacuna especifica, que proporcione protección efectiva a los grupos etarios de mayor riesgo. 


\section{RESUMEN}

Se presentan los hallazgos etiológicos determinados mediante bacteríología y prueba de aglutinación de látex, en 48 pacientes con meningitis bacteriana aguda, reunidos en un período de 3 años (julio 84 - julio 87). Los cultivos de LCR dieron resultados positivos en 31 pacientes $(64,6 \%)$, y la prueba de aglutinación de látex, realizada en 44 casos, dió una positividad global de $72,7 \%$, permitiendo aumentar el diagnóstico etiológico en 8 casos adicionales. $H$. influenzae fue el germen causal más frecuente: $61,5 \%$ con una mayor concentración de casos, en los lactantes menores, entre 3 y 12 meses de edad. St. pneumoniae se identificó en $20,5 \%$ de los casos y N. meningitidis: $12,8 \%$. La tincion de Gram tuvo un alto rendimiento $(83,3 \%)$ y mostró una estrecha correlación con la prueba de látex. El estudio de susceptibilidad a antimicrobianos, permitió detectar 3 cepas de $H$. influenzae con resistencia simultánea a ampicilina y cloramfenicol y un $100 \%$ de susceptibilidad a cefotaxima. No se verifico resistencia para el resto de las cepas de Haemophilus, a través de estudio de susceptibilidad por difusión en placa, como tampoco para cepas de St. pneumonize y $N$. meningitidis.

\section{REFERENCLAS}

1. Bolon G., Borzo M.: Acute bacterial meningitis in children and adults. A perspective. Med Clin North Am, 1985; 69: 231-241.

2. Lein J.. Feigin R., McCracken G. Jr.: Diagnosis and managcment of meningitis. Pediatrics (Suppkment Part. 2) 1986; 78: 959-982.

3. Dajani A., Asmar B., Thinumoorthi M.C.: Systemic Hacmophilus Influenzae disease: An overview. J Pediatr 1979; 94: 335-364.

4. Herrera P., Prenzel I., Arribas R., Rolón R. Topelberg $S$, : Haemophilus Influenzae en meningitis bacteriana aguda. Pediatria $1975 ; 18: 89-91$.

5. Herrere P., Prenzel I. Topelberg S.: Infecciones graves por Hemophilus Influenzae en el niño. A spectos generales y aspecto clínico. Rev Med Chile $1983 ; 111$ : 808-814.

6. Spread of Haemophilus Influenzae Ty pe b. Lancet. $1981 ; 1 ; 649$.

7. Band J., Froser D., Ajello G.: Prevention of Haemophilus Influenzae type b disease. JAMA. 1984; 25 1: 2381-2386.

8. Schlech W., Ward J., Band J., Hightower A., Fraser $D$., Broome $C$.: Bacterial Meningitis in 'the United States, 1978 Through 1981. JAMA 1985; 253: 1749-1754.

9. Ward J., Lum M., Halla B., Silimperi D., Bender $T$. Invasive Haemophilus Influenzae type b disease in Alaska: Backgroung Epidemiology for a vacune efficacy trial. J Infect Dis 1986; 153: 17-26.

10. Juliet $C$., Rodriguez $G$, Marti A., Burgos $O .:$ Menirgitis bacteriana en el niño: Experiencia con 441 casos. Rev Med Chile 1983; 111: 690-698.

11. Smith H.: Chemoprofilaxis of Meningitis. Arch Dis Child 1986;61:4.5.

12. Reyes L., Prado V., Sin M.T.: Perfil de sensibilidad a los antimicrobianos de Hemophilus Influenzae aislatos de patología infantil. Rev. Chil Pedjats $1984 ; 55: 54-56$.

13. Reyes L., Prodo V.: Tres años de vigilancia de la sensibilidad in vitro de Hemophilus Influenzae causante de infecciones pediátricas. Rev Chil Pediatr $1986 ; 57: 128-133$.

14. Lederman W.. Cohen J., Siri M.T., Banfi A.: Prevalencia de Hemophilus productores de betalactama sa en infecciones pediátricas. Rev Chil Pediatr 1983; 54: 166.

15. Whittle H.C., Tugwell P., Egler L.J., Greenwood B.M.: Rapid bacteriological diagnosis of pyogenic me ningitis by latex agglutination. Lance $1974 ; 2$ : $619-621$.

16. Severin W.P.J.: Latex apglutination in the diagnosis of meningococcal meningitis. J Clin Pathol 1972; 25: $1079-1082$.

17. Ward J., Siber G., Scheifele D., Smith D.: Rapid diagnosis of Hemophilus Influenzae type $b$ infections by latex particle aglutination and counterinmunoelectrophoresis. J Pediatr 1978; 93: 37-42.

18. Bortohissi R., Wort A., Casey S.: The latex agglutination test versus counterinmunoelectrophoresis for rapid diagnosis of bacterial meningitis. Can Med Assoc J 1982: 127: 489-493.

19. Newman $R$., Stevens $R$., Gafor $H$.: Latex agglutination test for the diagnosis of Haemophilus Influenzae meningitis. J Lab Clin Med 1970; 76: 107.113.

20. Kaplan S: Antigen detection in cerobrospinal Fluid-Pros and Cons Am J Med 1983; 109-118.

21. Dirks S., Zanen H.C.: Latex agglutination, counterinmunoelectrophoresis and protein A co-agglutination in diagnosis of bacterial meningitis. J Clin Pathol 1978; 31: 1167-1171.

22. Comercial Kits for bacteriological diagnosis of meningitis. Lancet $1984 ; 2$ : 500-501.

23. Del Valle M., Hanning S., Vial P., Montiel F., Cerda M.: Identificación rápida del agente etiológico médiante prueba de aglutinación de látex en meningitis bacteriana aguda. Rev Chil Pediatr 1985; 56: $159-161$.

24. Misraji A., Villarroel J., Del Canto, et al: Experiencia preliminar con la prueba de aglutinación de látex en la detección precoz de Haemophilus Influenzae tipo b en meningitis aguda bacteriana. Rev Chil Infect 1985;2:61-64.

25. Robson H.G., Salit l.E.: Susceptibility of Neisseria Gonortheae to seven antibiotics in vitro. Can Med Assoc J 1972; 107: 959-962.

26. Gratten M., Barker J., Shann F., et al: Non type b Haemophilus Influenzae meningitis. Lancet 1985; 1: 1343-1344.

27. Campos J., Garcia-Tomel S., Gairi J., Fabregues I.: Multiply resistant Haemophilus Influenzae type $b$ causing meningitis: Comparative cinical and taboratory study. J Pediatt 1986; 108: 897-902. 
28. Montiel F., Lobos T., Acuña G., Lam M.: Breviario de la terapia antimictobiana. Stgo Fac Med, Universidad Católica de Chile 1987; 7 .

29. Anhalt J.P., Sabath L.D., Barry A.L.: Pruebas espe- ciales: deteccion de producción de betalactamasa Lenette, Edwing $H$. Manual de Microbiología Cínica. 3a. Ed. Buenos Aires. Panamericana S.A. $1982 ; 585+586$. 